\title{
Sub-matter Higgs Condensate in Discrete Bounded Fields
}

\author{
Peter A. Jackson ${ }^{1 *}$, John S. Minkowski ${ }^{2}$ \\ ${ }^{1}$ Hillside Lab, Orchard House, Hillside Road, Whitstable Canterbury Kent, CT5 3EX, UK \\ ${ }^{2}$ Assistant Professor (Retired), Johns Hopkins University, Baltimore, Maryland, USA \\ Email: pj.ukc.edu@physics.org
}

\begin{abstract}
We propose a 'sub-matter' scale 'Higgs condensate' from which pair production and 'action-at-a-distance', inherent in Electromagnetism and Gravity, emerge. Disturbances cause larger, fermion scale, rotations which can only then couple with Electromagnetic scale field fluctuations. Re-emissions at $\mathrm{c}$ in each fermion's centre-of-mass rest frame, give local c at all electron/positron 'fine structure' (/plasma $\mathrm{n}=1$ ). Fermion translation in backgrounds increases condensation and rotates optical axes producing aberration of light as Kinetic Reverse Refraction. The Higgs condensate cannot 'couple with' electromagnetic waves, like water waves of particles, so is not 'luminiferous aether'. It has mobility, but energy density variations disappear at large scales, where no matter exists, or after polarity 'annihilations'. It's consistent with 'dark matter', the Casimir force, and 'curled-up' dimensions. Charge alignment 'standing waves' provide Faraday's electromagnetic field interactions. Density distribution around condensed matter maintains pressure gradient at the inverse-square of distance reproducing 'gravity'. Other anomalous effects logically resolve.
\end{abstract}

Keywords: Higgs, condensate, dark matter, dark energy, relativity, quantum gravity, electromagnetism.

\section{Introduction}

Implied 'action-at-a-distance' and the related 'luminiferous aether' concept have long been recognised as problematic to physical theory. Newton knew gravity needed something, tried but rejected 'aether' and accepted his 'mathematical' solution would have to do. Boscovich postulated sub-atomic 'point' particles as a 'new' form of aether, as later did Dirac. Maxwell relied on it, Faraday shaped and aligned it, both without resolving it. Minkowski said; "everywhere and everywhen there is something perceptible...I will use...the word substance" as "endlessly many spaces;" which "at any world-point may always, ...be looked upon as at rest." (Cologne 1908). Einstein said; "space without aether is unthinkable' (Leyden 1920) and; "it must now be remembered that there is an infinite number of spaces, which are in motion with respect to each other." and such spaces are; "not thought of as bounded, and to assume that these two spaces are in motion with respect to each other." (Relativity. 5th Ed.1952)[1], but he never found the physical analogue or 'boundary' to resolve the impasse, we suggest because space travel came too late. We now invoke the two-fluid plasma (Shumlak 2004)[2] of astrophysical 'shocks' condensed around all matter in motion as forming those 'boundaries' defining the 'Higgs condensate' (HC) model of 'discrete fields' (DFM) or Einstein "spaces..in motion within spaces."

Direct detection via Electromagnetism (EM) has a lower scale limited by minimum wavelength gamma and by minimum condensed 'particle size'. Below condensed particles no lower size limit need exist. There is wide inferred evidence of sub-matter 'dimensions', and a Higgs vacuum expectation value (VEV). We hypothesise a 'next scale down' state, which may be visualised as dense clouds of a submatter energetic particle condensate. As in water, the motion of medium particles is very different to that of 'wave' fluctuations. We give some characteristics for this 'Higgs condensate' which provide rationalisation for many effects not explainable at larger scales. The Casimir force between 2 plates in a vacuum was predicted in 1948 and confirmed in 1998, given by $F(a)=-K / a 4$, where $\mathrm{K}=2 h c / 240=$ $1.3 \times 10^{-27} \mathrm{Nm}^{2}, h$ is Planck's constant, c is the speed of light in vacuum. The small force still gives a high vacuum energy density $\left(\sim 10^{114}\right.$ Joules $/ \mathrm{m} 3$ or, in terms of mass, $\left.10^{95} \mathrm{~g} / \mathrm{cm}^{3}\right)$ if using a Planck length $10^{-35} \mathrm{~m}$ scale cut off, making no physical sense in General Relativity (GR) and problematic for 'spacetime' and cosmological constant $\Lambda$ so largely ignored. Astronomical data suggest $\Lambda$ might be of the order 
$\sim 4 \mathrm{eV} / \mathrm{mm}^{3}$, or a mass of $10^{-29} \mathrm{~g} / \mathrm{cm}^{3}$. Leading Casimir and Quantum Electrodynamics (QED) Prof. J. Maclay found the discrepancy "between theory and observation is about 120 orders of magnitude.. the greatest quantitative discrepancy between theory and observation in the history of science." [3] No consistent interpretation of this 'zero-point energy density' in space exists to settle this conflict between 'well verified theories'; QED and General Relativity. Other theorists have tended to avoid addressing the issue. Maclay also notes that rigorous QED calculations "are very difficult and have yet to be completed. " [4]

The HC and resultant DFM suggest some different fundamental assumptions should be tested. Removing $10^{-35}$ as an 'ultimate' lowest scale cut off is proposed. Once changed then little more than interpretive changes can resolve the apparent inconsistencies.

The question "what IS matter?" has been beyond physics. We first assume it as the additional 'rotation' of the Higgs process. The answer to 'what is rotating?' is then simply 'smaller rotations', the first step of possibly infinite Gödel or Superstring Theory's 'dimensional' recursion. Vortices, spherical and complex rotations are self contained 'quanta' which can propagate with unique linear (relative) motions. The property of self sustained infinite repetition produces a finite 'energy' within a finite space. The electron rotational energy we find should be constant, but relative speed gives us the higher energies.

Linear propagation gives the helical charge paths familiar in photonics; opposing charges give the twin helix. Rotations on the minor axes give ellipticity. The twin vortex torus closes to a spheroid describing the form of a magnetic field or galactic halo. For simplicity we consider our sub-matter HC 'entity' as defined by a spherical rotation so with spheroidal limits. Klien's 'radius' (though not 'circles') of $\sim 10^{-}$ ${ }^{30} \mathrm{~cm}$ may be valid but in common with string theory no limit on dimensions, so no Planck 'hard deck' scale, emerges. The HC differs from Kaluza-Klien (KK) theory's 5th 'curled up' or 'compact space' dimension (Wesson 2006) [5] as the gravitational constant is not seen as an EM coupling constant in the metric. Also group action is allowed, so producing Chiral fermions to couple by absorption / reemission. Gravitational potential is then a distribution of HC 'pressure/ density' maintained by fermion spin.

The HC is not continuous or immobile as the (Thomson etc.) 1867 'Vortex-aether'. There is a 'step down' in scale or 'dimension' which may be seen as one fractal order or visualised as the scale difference between a 'dust devil' or cyclone and the 'particles' orbiting to form one. For an initial proof of concept model of the $\mathrm{HC}$ mathematical precision is not sought; indeed many effects require the irreducible Stokes-Navier magneto-hydrodynamic (MHD) 3-dimensional viscosity extension to the Euler equation for moving 'fluids', which has (as yet) no exact solution. We also find that trying to 'understand' nature by mathematics alone has left much 'hidden' from perception. We here take the Einstein/Wheeler view of physical analysis preceding mathematical modeling. The $\mathrm{HC}$ need not imply a 'dark energy' accelerating expansion of the universe, propagation at $>c$, or act as 'space-time' or 'aether'. It will however enable the effects attributed to those and similar concepts and descriptions to be physically derived. More coherent understanding of the CMB 'temperature' of $0.2348 \mathrm{meV}(2.7250 \mathrm{~K})$ and so called Zero Point Energy can also emerge. The paper reviews and analyzes effects using three general theory sections; EM, Gravity and Quantum Mechanics (QM), plus a summary, relying on minimal other assumptions, while using the simplest physical approach and non-technical language possible.

\section{$2 \quad$ Electromagnetism}

Charged particles influence others 'at a distance', and Faraday's field lines still lack physical form. The $\mathrm{HC}$ fills all space so provides an independent 'transmission' substance. If equivalent to a fermion field, the $\mathrm{HC}$ rotational poles will align with the curving field lines from pole to pole. A polar 'topology' then extends well beyond the fermion radius, interacting with other particle fields so influencing particle dynamics. Relative linear motion between particles and accompanying HC fields will intensify influences. Further research will be required to establish if the HC elements 'flow along' the field lines, so physically interact with the massive particles.

If $+/$ - charge polarity derives from polar rotation, then flipping an electron, or looking towards the opposite (south) pole, will reveal a positron. Both spherical and toroidal anticlockwise (-) AND clockwise $(+)$ polar rotations exist for rotating bodies at all scales. Either may then represent simple 
equivalence to Chiral Majorana fermions (its own 'antiparticle'). Direction of 'view' OR motion on or along a polar axis vector is then critical as only one polarity can ever be detected at a time. 'Polarity wave' propagation then rotates polar axes in the HC.

Recent PAMELA data confirmed NO electron excess but equal positron content at measured energies (Adriani O., et al.) [6] For spherical rotation changes in 'surface' charge speed/momentum amplitude will vary by the cosine of the 'angle of latitude'. Such change rates of momentum if exchanged on interactions, which we consider as 'measurement', have wider implications including in QM (See 5. below).

Gyroscopics is also not fully resolved. Rotation and precession seem to modify gravitational effects on spinning bodies. If gravitating matter is itself 'motion' then HC field distortions from other motion will naturally influence local gravitational potentials and vectors producing the gyroscopic effects found.

Fast spinning discs have been found to increase in 'weight' many orders greater than predicted in GR's gravitomagnetic field, (i.e. Tajmar \& de Matos 2006) [7], and rapidly spinning toroidal active galactic nuclei (AGN) are found to generate turbulent gravitational potentials (Yang et al 2015) [8] both effects are implicit in our proposed model.

Comet Coma explanations have been problematic, as has the 'Unruh Effect' (i.e. Crispino et al 2008) [9] attempting to explain electron fine structure around matter in motion. The 'dirty snowball' model was very different to the solid objects found. The HC mechanism naturally produces coma as plasma shock propagation related to speed in the ambient medium or solar wind frame. The fine structure constant $\alpha\left(=\mathrm{e}^{2} / \hbar c\right)$ may then be seen as the ratio of electron to photon speeds $\alpha=\mathrm{e}^{2} /\left(2 \varepsilon_{\mathrm{o}} h c\right)=\mathrm{v}_{\mathrm{e}} / \mathrm{c}$ $=1 / 137.036$ of Sohrab \& Siavash (2015) [10] who give mean thermal speed of the electron at equilibrium with photon gas as $\mathrm{V}_{\mathrm{e}}=2.187640 \times 10^{6} \mathrm{~m} / \mathrm{s}$ and its de Broglie wavelength as $\lambda_{\mathrm{e}}=3.3250 \times 10^{-10} \mathrm{~m}$.

\section{Sub-matter Quantum Gravity}

The HC density distribution varies from the large scale behaviour of gas by maintaining the 3D density or 'pressure gradient' distribution reducing by the square of the distance from the gravitating rotation, or of the complex set of rotations forming bound molecular particles and all more 'massive' bodies. However we invoke Euler, whose equations lead to the Stokes-Navier Magnetohydrodynamics (MHD), studied under and built on the work of Bernoulli, who gave the key relationship between motion and pressure reduction. We experience this relation in atmospheric behaviour on Earth and in the negative pressure of accelerated airflow which powers sailing yachts. Euler's work was a precursor of string theory, agreeing with at least the HC's first higher dimensional order motions and oscillations, as well as further smaller 'dimensions'.

Pressure reduction from relative motion might imply either an $\mathrm{HC}$ in constant but graded orbital motion around the gravitating body or a varying spin speed of the $\mathrm{HC}$ elements themselves. Both options may complicate other effects and further investigation is needed to determine detailed causal dynamics. Whatever details emerge, the overall 'density gradient' topology can now be created and maintained in the sub-quantum HC similar to the popular 'Mexican Hat' model. The maintained gradient and (at large scales) flat overall ground state obeys the law of conservation of total 'angular momentum' energy. Each 'nearby' body then experiences asymmetry of HC pressure so is affected by and drawn towards the other in proportion to combined total spin energy. Changes in the topography propagate at $\mathrm{c}$ from the gravitating body, in which case no need for graviton 'messenger particles' arises. The Mexican hat simply flattens when the central peak stops rotating.

The 'inverse square law' approximation of Newton has a constant relationship to the relative surface area of the sphere at any radius; $4 \mathrm{p} \mathrm{r}^{2}$, The spherical surface in turn has a direct relationship to the density, or 'intensity', of the expanding Schrödinger sphere 'causal' surface potentials propagating radially, considered as a 'plane wave' at large radii. We suggest it should then seem quite natural that an $\mathrm{HC}$ density distribution change would be directly related to $\mathrm{r}^{2}$.

Another interesting consequence of our definition of condensed 'matter' as rotational energy is that the rotational and (squared 'relative') linear momentum add up to the total energy exchanged on interaction with any system (as 'measurement'). The equation $\boldsymbol{e}=\boldsymbol{m} \boldsymbol{c}^{2}$ can then simply describe the energy of a spinning ball thrown by a rugby player or quarterback found by a receiver. The more spin the more energy found, but the speed of the ball, including the receiver's speed towards the passer 
squares the amplitude of the inherent (three dimensional so two orthogonal component) spin giving the total 'inertial mass' constituting the ball.

On the topic of gravity we note that the ubiquitous $n=1$ fermion pairs found ever more commonly as 'space plasma' are likely to make a significant contribution to the density of 'dark matter' haloes around galaxies and all rotating celestial bodies, including Earth's familiar dense Plasmasphere $\left(<10^{14} / \mathrm{cm}^{-3}\right)$, beyond our spheroidal atmosphere in our toroidal EM field. Okada \& Yamada (2013) [11] and Ho \& Scherrer (2013) [12] identify simple or toroidal Higgs based Majorana fermion dark matter models. The Voyager probes encountered high plasma density in the heliosheath ('shock') and even since 2013 find electron/positron (plasma) densities of $\sim 0.1 / \mathrm{cm}^{-3}$ in the interstellar medium (ISM) of the galaxy.

Halo density estimates have been lower $\left(\sim 0.035 / \mathrm{cm}^{-3}\right)$ but increasing. Galactic H1 mass alone was estimated by Henderson, Jackson \& Kerr (1982) [13] to be $4.81 \times 10^{9}$ solar masses, $81 \%$ of that beyond $\mathrm{R}=11 \mathrm{kpc}$. Current standard Halo models consider galaxies as embedded in isothermal dark matter haloes described by a Maxwell-Boltzmann phase-space distribution.

Planck probe data led to a $13 \%$ jump in Local Group mass estimates including dark matter and energy (Partridge, Lahav, Hoffman, 2017) [14] in agreement with recent predictions, i.e. Chernin et al (2009). [15] Most reported studies give galactic dark matter densities in the energy range 0.2-0.6 $\mathrm{GeV} / \mathrm{cm} 3$ with a $270 \mathrm{~km} / \mathrm{s}$ velocity dispersion. An electron's non-zero inertial and thus gravitational mass renders it reasonable to suggest that the apparently 'anomalous' gravitational mass of galaxies may prove attributable to non-exotic particles condensed from the $\mathrm{HC}$ at the boundary transition zones (TZ's) between kinetic states.

We've 'sketched out' the dynamic of constant local c implicit in the HC discrete spaces or bounded sub-quantum 'fields' model (DFM). Nature tends to do nothing without good reason. The free electron shocks propagated at the boundaries between 'states of motion' do so proportionally to relative $\mathrm{v}$ giving the fermion density required to effect the speed change to maintain local c in the next field. The energy is of shear plane or pressure differential. Consistent with data, if not in any theory, we postulate that: All electrons re-emit absorbed EM fluctuations at c LOCALLY in each electron's centre-of-mass rest frame.

In diffuse electron media (i.e. the Interstellar medium) the long established (but ill fitting current theories) Ewald-Oseen extinction distance (i.e. Ballenegger 1999) [16] increases up to many parsecs, with Nobelist Sir Chandrasekhara Raman's scattering and bi- or multi-refringence evident during the process (Raman 1922) [17] all as J.D. Jackson's definitive text 'Electrodynamics'. At the refractive plane of a moving lens the extinction distance is $<1$ micron. We identify this distance as Maxwell's kinetic 'Near/Far field transition zone' (TZ) never theoretically rationalised, where Snell's law of refraction fails. Problematic 'Fraunhoffer' refraction/radiation takes over in the 'far' field, which the DFM rationalises and identifies as quantified by the algorithms of Kinetic reverse refraction (see below). H.A Lorentz developed his transformation (the 'LT') from previous work including Maxwell's TZ.

\subsection{Non-'collisionless' Boundary Shocks}

We have elsewhere discussed (Jackson, 2013) [18] an implicit non-linear physical process as propagation $\mathrm{v}$ nears c and plasma density nears 'optical breakdown' (OB) $\sim 10^{23} / \mathrm{cm} /{ }^{-3}$ which the non-linear LT curve closely models. Heat increases and oscillation is restricted non-linearly. In the Large Hadron Collider (LHC) tube the dense cloud of 'photo'-electrons condensed in both the bunch and pipe rest frames are now termed 'virtual' to address the apparent conservation issue, but the LT curve is closely reproduced in the LHC power input curve. The high energy electrons fill the pipe and damage the pipe wall. Astrophysical shocks, seen as "collisionless", are orientated to both solar wind and orbital path vectors. Earth's complex MHD shock 'electron' turbulence leads into a streaming magnetotail. (see Fig. 1) 


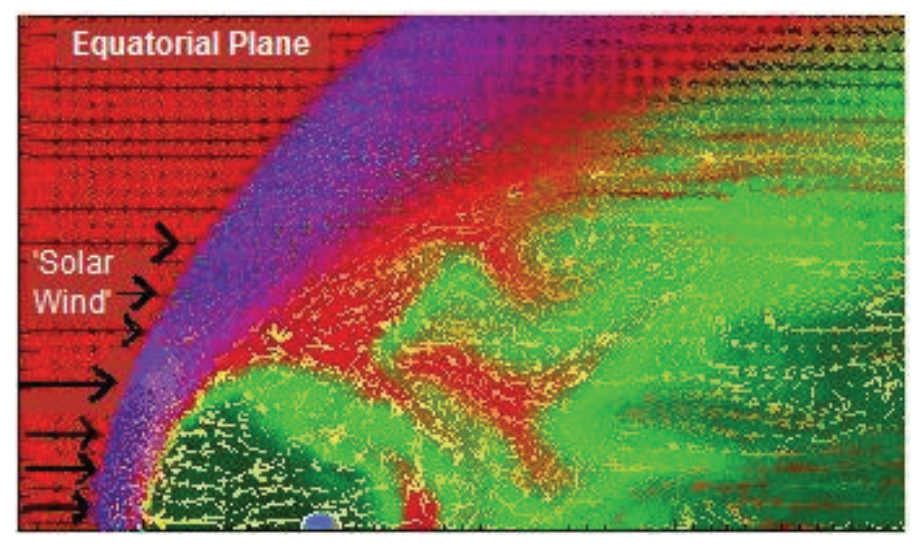

Figure 1. Earth's Bow Shock. Magnetohydrodynamic (MHD) turbulence vectors. Note the implied but unrecognised speed change we identify at the shock crossing. (NASA)

Findings have shown the plasma physics work of Nobel Laureate Hannes Alfven and many others is consistent with, but remains peripheral to, theoretical analysis. Data from the multi ('Cluster') probe missions across the shock and magnetotail has not allowed logical rationalisation under current assumptions, suggesting some are very likely to be flawed or false.

Head of the UK's Imperial College section specialising in analysis of Earth's bow shock and magnetotail, Steven Schwartz, has stated; “...the electron heating and smaller scale fields remain poorly understood and controversial." (2011) [19]. (See also Fig. 2 below.)

The DFM suggests the "collisionless" concept and bare 'electron temperature' descriptions are flawed and incomplete. Detected energy levels are rooted in both rotational and relative linear motions. 'Field strength' has contributions from 'density' as well as lambda (and so inversely f), Doppler shifted towards Gamma at the shock compression zone. The boundary collision of the two systems in different rest frames, described by Shumlaks two-fluid plasma algorithms, will then create the step in energy level across the boundary zone implicit in the Cluster data.

On each electron's strong coupling with EM an angular velocity of re-emission at $2.04977 \times 10^{22} \mathrm{rads}=\mathrm{c}$. EM propagation speed is then firmly tied to each electron centre-of-mass or LOCAL field rest frame. No 'mystery' would then remain about the constant speed of light, or shock crossings. (See Fig. 2 below).

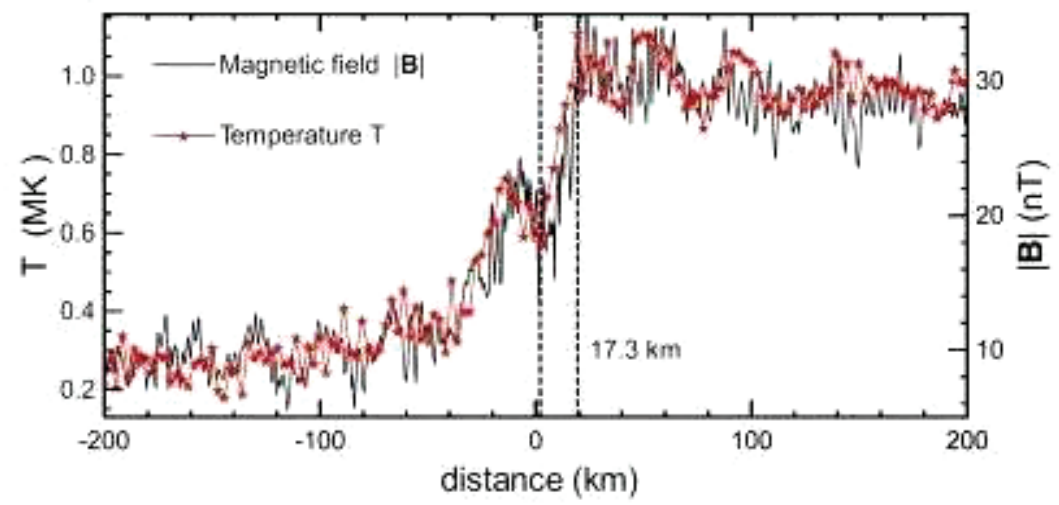

Figure 2. NASA 'Cluster' data plot. S. Schwartz Imperial Coll. Change in electron density, EM wavelength and $f$ at Earth's Bow Shock; described as the plasma 'temperature surrounding' the shock. Again rationalised here as a 'speed' / IRF change'.

The key problem we identify is poor understanding of 'LOCAL' c, so implication of speed change required to maintain c locally. 'Speed' is an entirely 'relative' concept. The Earth's 'system' rest frame moves at $30 \mathrm{~km} / \mathrm{sec}$ with respect to the solar system (Barycentric) rest frame and faster still with 
respect to the 'solar wind'. We identify a 'hidden factor' here; a real speed change, so Doppler shift of light entering a new medium IRF when the media systems are in relative motion. (giving a change between Einstein 'kinetic states' i.e. k to k'). That speed change at the shock produces the Fig. 2 data, and is dynamically consistent with George Stokes 'Dragged Aether' theory, (i.e. see Mineur 1927) [20] but free of the old 'aether' and its problems!

Stokes' popular model overcame problems with Newton's 'single aether state', but was discredited in error by Lodge's (1892-93) [21][22] 'spinning glass disc' light path analysis. Lodge analysed the 'ray' angle from his lab frame 'dragged' by the disc, but the correct (local) frame representing Earth's atmosphere was that of the glass, from where the Poynting vector is reversed, giving the true 'stellar aberration' effect. The error, never corrected and later repeated, i.e. Jones [23] is analysed in Jackson, Nixey (2012).[24]. The course of physics was changed as all including Lorentz and Einstein thought Stokes hypothesis had been falsified. Stokes anyway found the 'localisation' mechanism for the speed and optical axis changes produced by the quite new concept of re-emissions at $c$ with respect to each electron's own centre-of-mass rest frame. A step closer to this model was 'Extinction Shift' theory, having similar dynamics but treating gravity differently. (NASA's E.H. Dowdye Jr. 2007). [25]

Critical differences between the observation data from each frame were shown by Huang (1994) [26] also in Gjurchinovski's 'HFP', (2004) [27]. There is a significant dynamic refraction effect of plasma clouds in motion localising light propagation speed and rotating the optical axis (changing apparent source positions). The effects have been confirmed by the very long baseline array (VLBA) findings (Pushkarev et al. 2013).[28] We've importantly identified that optical axes (giving apparent source position) cannot remain on wavefront normals after such kinetically induced rotation (Jackson 2012).[29]

Difficult to accept so far are the implications of this confirmation for Special Relativity (SR) which may be profound as Stellar Aberration predictions still lack a consistent relativistic algorithm, corrections 'by hand' still being needed. (see USNO Circ.172. p6) [30] Theoretical rationalisation does then emerge using optical axis rotation and 'Kinetic Reverse Refraction' (KRR). Figure 3 below shows the geometric effect of the algorithms. For SR the 'physical' rationale Einstein sought emerges. (also see Mathpages) [31]

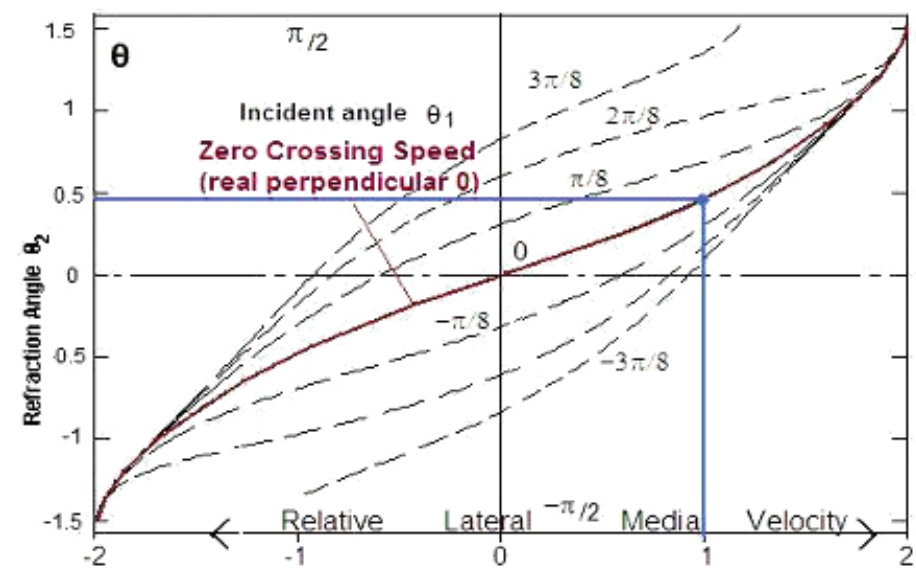

Figure 3. Refraction at a plane boundary between moving media (KRR). See also Inverse Compton scattering and the kinetic Sunyaev-Zel'dovich effect.

Using Einstein's 'heuristic' terms we may consider the 2-fluid plasma crossing as analogous to the fluid 'torque converter' drive coupling of a car automatic gearbox. The input shaft has one speed, and output shaft another. The viscous fluid has hydrodynamic turbulence between. The speed change is dependent on viscosity, or on 'density'. In the DFM the speed change across the electron shock is principally linear and not rotational. Even simpler 'thought experiments' are possible:

1. A simple glass box or block is moving in space at v. Light, propagating at local c, has no idea the block even exists until arrival. Now we know all light WITHIN the glass and/or the box! propagates at $\mathrm{c} / \mathrm{n}$ with respect to the GLASS rest frame. Logic then dictates that not only does the light change 
speed to conform to the refractive index $\mathrm{n}$ of the glass, but a second speed change is by $\mathrm{v}$, the speed of the glass block in the local background rest frame, so maintaining $\mathrm{c} / \mathrm{n}$ in the new 'frame'.

2. Observe a sprinter doing a 10 second $100 \mathrm{~m}$ dash on a train doing v. An observer on the platform may time him at a new record pace! SR's maths attempt to prevent that. However the maths are not required. The record pace, or apparent $\mathrm{c}+\mathrm{v}$ photon pulse, is not a 'propagation speed'. It is only an apparent rate of angular displacement measurable from a series of lateral emissions propagating at $\mathrm{c}$.

Einstein identified that clear logic in his "...infinite number of spaces in motion relatively to each other.. .. not thought of as bounded," ... "This latter idea is indeed logically unavoidable, but is far from having played a considerable rôle even in scientific thought." (1952) [1] Such a concept can now be rationalised using the two-fluid plasma boundaries we propose at Maxwell's Near/Far field transition zones. The idea may then play that "considerable role ... in scientific thought".

No experimental proof of KK or string theory has been possible as we 'use' EM to observe, but most of our 'knowledge' about nature is based on inference and logical consistency in resolving anomalies. No coherent logic for EM or gravitational action-at-a-distance exists so we suggest any model producing both may advance understanding, even at the cost of further 'cutting' of Democritus's 'indivisible' atom.

\section{Quantum Non-Locality}

Einstein objected to the apparent 'superluminal communication' of the quantum theoretic interpretation of statistical data. He argued directly with Bohr calling it "spooky" at the 1927 Solvay conference, the resultant (with Podolski \& Rosen) 'EPR paradox' remains unresolved. Wide alternatives to the violation of SR's light postulate (propagation $<\mathrm{c}$ ) have been proposed. Our HC model appears consistent with none of them! The process described does however suggest that, on using modified starting assumptions, including both Maxwell's momenta, (as a Poincare sphere) a mechanistic sequence violating Bell's inequality allows a new interpretation of the statistics, shockingly perhaps not reliant on 'action-at-adistance'! (or so called 'hidden variables').

We have identified above that for spherical rotation changes in polar 'charge' and surface speed (momentum) amplitudes vary inversely by the cosine of the 'angle of latitude'. Speed change rates are known in geometry and geophysics but Maxwell's inverse orthogonal rotational 'curl' momentum is as bounded fields in motion; "far from having played a considerable rôle even in scientific thought." (Ed. 5. 1952) [1]

Detector A or B 'polariser/modulator' field electrons will carry that inverse PAIR of momenta, which on 'absorption' interaction with a similar ARRIVING particle (or 'state') will 're-emit' a combined (from vector addition) amplitude of cosine values dependent on the RELATIVE polar (x) axis angle (for ANY $y$ or $z$ axes orientations. The next part of the sequence repeats the process at the photomultiplier (Pm, or a P-'diode'), applying a second Cos Lat. amplitude distribution, dictating output 'click' rates for any orthogonal orientations (with no click below a minimum threshold amplitude zone near zero $+/$ - amplitudes). The result then is that if A (or B) reverse their detector field angle setting just before arrival, their own finding is reversed. The same 50:50 data is produced but the assumption of 'how' differs from the QM interpretation, which implies A must reverse $B$ 's finding, even at a distance.

\subsection{Entanglement}

'Entanglement' in this case only requires the maintained anti-parallel polar axis of each pair 'north' (-) pole one end, 'south' (+) pole the other. The mechanisms described may or may not closely model nature but subject to the above physical processes and conditions being met, the output data violate Bell's inequality and obey the Dirac equation. The mysterious ' $\mathrm{Cos}^{2}$ ' curves from 'click' rates emerge from the $\boldsymbol{t} \boldsymbol{w o}$ applications in series of cosine value amplitude vector additions, or the 'intensity' of Malus' Law (Intensity (I) $\infty \cos ^{2} \theta$ ) It should be noted that the suggested model is not a 'local hidden variable theory" and is in agreement with John Bell's own idea of where the logical solution "requiring an imaginative leap that will astonish us" may lie, which was in 'fermion number density'. (J Bell, 1987). [32],[33] 
The new starting assumption is the surface vector distributions of the Poincare sphere (opposing curls near poles going to zero at the equator, inverse to linear amplitude, measured orthogonally). The two inverse states, both then reversed at $180^{\circ}$ degrees, represent QM's unexplained 'superposed states'. (Bohr cautiously assumed NO 'starting morphology' for split pairs). It is those momentum state amplitudes varying with angle which constitute 'measurements', which cannot both be detected with certainty at the same time, i.e. measuring $+/$ - polarity precisely on the equator will give 50:50 results (max uncertainty) as will up/down (or 'left/right') linear motion at either pole.

\subsection{Uncertainty}

The Bayesian 'distribution' description of quantum uncertainty then has validity. Born's strict 'probability wave' interpretation of the wave equation to which Schrödinger objected becomes unnecessary if the 'wave' is, like nature, a 3D phenomena. Orbital motion on all THREE axes while translating describes the helical (with ellipticity) charge paths so closely akin to Euler's equations and thus to 'cosmic strings'. As rotational momentum, (as surface 'speed') so also likely momentum exchange between rotating spheres ('measurement'), varies by Cos theta latitude, $\left(0-90^{\circ}\right)$ with Maxwell's 'curl' changing inversely with linear (equator) momentum; we can then use vector addition on the 3 $(\mathrm{x}, \mathrm{y}, \mathrm{z})$ rotational axes to obtain measure amplitudes. Doing so at both polariser and photomultiplier gives the square of the Cos values, reproducing the Dirac QM equation.

While mechanistically simple to verify, readers shouldn't be concerned if unable to assess this derivation as a good knowledge of quantum experimental outcomes is needed without being wedded to interpretation. As QM is so complex we don't attempt a full analysis here. We $\boldsymbol{d o}$ invite specialists in the field to test and falsify using our different starting assumptions, also the fuller explanation and confirmation experiment discussed here (FQXi, 2018) [34] and an independent computer plot verification of an equivalent code by (Traill, 2018). [35]

\section{Summary}

We propose a sub-matter scale Higgs condensate medium, which transports but cannot 'couple with' EM's fluctuation scale, so is not a 'luminiferous aether'. (see Fig. 4). Condensation due to perturbation provides the electron positron pairs which couple strongly so forming physical two-fluid plasma 'boundaries' between mobile regions of the HC. Electron spin speed then controls and localises propagation speed c on re-emission. The Minkowski/Einstein "Infinitely many regions of space" emerge, but now as discrete "bounded" fields.

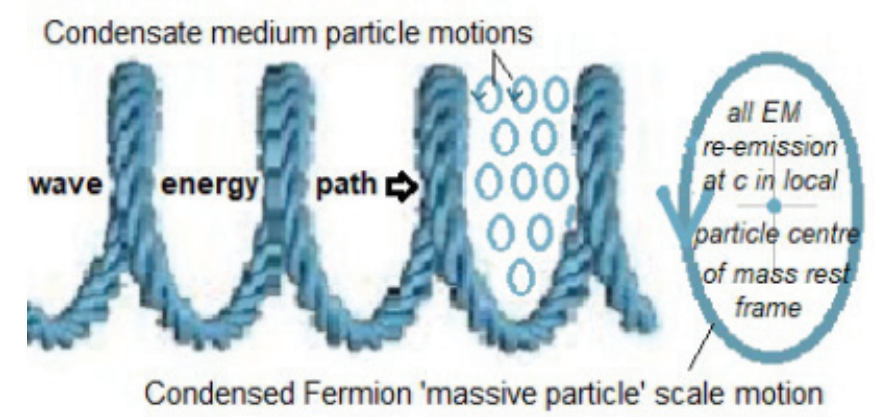

Figure 4. Scale distinction between wave motion and particle motions in media. Particles formed can only couple with passing waves via larger scale rotations. Note also that 'rope' is made up purely of many recursive scales of helical strands.

An early prediction of sub-matter, so sub-EM scale structure came from Thomson at a Johns Hopkins Lecture in 1884; "It may be found in some future time that aether too has some molecular structure, perhaps much finer than any structure of ponderable matter;" [36]. The Higgs Condensate meets the identified needs performing three other key functions: 
1. Maintain a density gradient around the 'spin' of condensed matter, so producing 'gravitation'.

2. Provide the medium for all 'action-at-a-distance' of EM potential between charges using its three (axes) degrees of rotational freedom plus 'elasticity'.

3. Allows the condensation of matter fine structure at kinetic system interfaces (shocks and refractive planes at Maxwell's near/far field boundaries) to interact with and constantly localize the speed of light c, consistent with Einstein's postulates if not with their common interpretation.

The HC model does not allow 'non-local' communication but does suggest that defining 'entanglement' as maintained 'split pair' polar axes allows exchange of momentum during detector electron interaction (surface rotation and speed vector additions) to produce orthogonal amplitudes and 'click rates' matching Dirac's QM equation. A new direction for studies in (sub-) quantum gravity with wider implications is thus suggested.

\section{References}

1. A. Einstein, "Appendix V, Relativity and the Problems of Space," (ed. 15, 1952), in Relativity: The Special and the General Theory, translation by R. W. Lawson. Crown Publishers, 1961, pp.135-157.

2. U. Shumlak, C. Aberle, A. Hakim, and J. Loverich, "Plasma Simulation Algorithm for the Two-Fluid Plasma Model," in Conference on Computational Physics, University of Washington, Seattle, 2004. Available: www.researchgate.net/publication/253755786_Plasma_Simulation_Algorithm_for_the_Two_Fluid_Plasma_ Model

3. G. J. Maclay, "Thrusting Against the Quantum Vacuum," March, 2007 in Frontiers of Propulsion in Science. AIAA, 2009, ch. 12, p. 6. Available: https://www.quantumfields.com/13_Maclay_Final1.pdf

4. G. J. Maclay, "The Role of Quantum Vacuum Forces in Microelectromechanical Systems." Sept 2006. Available: https://www.quantumfields.com/usesofvacuum.pdf, p. 5.

5. P. S. Wesson, Five-Dimensional Physics. World Scientific, 2006, pp. 2-24.

6. PAMELA Collaboration: O. Adriani et al., "The cosmic-ray positron energy spectrum measured by PAMELA," Physical Review Letters, vol. 111, 081102, 2013.

7. B. Malekolkalami and M. Farhoudi, "About Gravitomagnetism," Modern Physics Letters A, vol. 24, no. 8, pp. 601-613, 2009.

8. H. Yang, A. Zimmerman, and L. Lehner, "Turbulent Black Holes," Physical Review Letters, vol. 114, 081101, 2015. Available: https://www.researchgate.net/publication/260295015_Turbulent_Black_Holes

9. L. C. B. Crispino, A. Higuchi, and G. E. A. Matsas, "The Unruh effect and its applications," Reviews of Modern Physics, vol. 80, pp.787-838, 2008. Available: https://arxiv.org/abs/0710.5373

10. S. Sohrab, "A New Physical Meaning of Sommerfeld Fine Structure Constant," APS, April Meeting, 2015.

11. N. Okada and T. Yamada, "Simple fermionic dark matter models and Higgs boson couplings," Springer, JHEP 17, 2013. Available: https://arxiv.org/abs/1304.2962

12. C. M. Ho and R. J. Scherrer, "Anapole Dark Matter," Physics Letters, B722, 341, 2013.

13. A. P. Henderson, P. D. Jackson, and F. J. Kerr, "The distribution of neutral atomic hydrogen in our galaxy beyond the solar circle," Astrophysical Journal, vol. 263, part 1, pp. 116-122, 1982.

14. C. Partridge, O. Lahav, and Y. Hoffman. "Weighing the Local Group in the presence of dark energy," 2013. Available: http://mnrasl.oxfordjournals.org/content/early/2013/09/17/mnrasl.slt109.full.pdf

15. A. D. Chernin, P. Teerikorpi, M. J. Valtonen, V. P. Dolgachev, L. M. Domozhilova, and G. G. Byrd, "Local dark matter and dark energy as estimated on a scale of $\sim 1 \mathrm{Mpc}$ in a self-consistent way," $A \mathscr{E} A$, vol. 507, pp. 1271-1276, 2009. Available: https://www.aanda.org/articles/aa/full_html/2009/45/aa12762-09/aa12762-09.html

16. V. C. Ballenegger, "The Ewald-Oseen extinction theorem and extinction lengths," American Journal of Physics, vol. 67, p. $599,1999$.

17. C. V. Raman. "Molecular Diffraction of Light," University of Calcutta. Nobel prize winning paper. February, 1922. Available: https://archive.org/stream/moleculardiffrac00ramarich?ref=ol\#page/n6/mode/2up

18. P. A. Jackson, "Optical Breakdown limit as a Mechanism for the Lorentz Transformation," Academia.Edu, 3715747 v1, May, 2013. Available: http://viXra.org/abs/1306.0071

19. S. J. Schwartz et al., "Electron Temperature Gradient Scale at Collisionless Shocks" Physical Review Letters, vol. 107, 215002, 2011. 
20. H. Mineur, "The experiment of Miller and the hypothesis of the dragging along of the ether," Journal of the RAS of Canada, vol. 21, p. 206, 1927. Avail: http://articles.adsabs.harvard.edu/full/1927JRASC..21..206M/ 0000206.000.html

21. O. Lodge, "Aberration Problems," Philosophical Transactions of the Royal Society. 184, Fig. 13, p. 780, 1893.

22. O. Lodge, "Aberration Problems and the connexion between Ether and gross Matter," March, 1892, Philosophical Transactions, Series A, p. 777, 1893.

23. R.V. Jones, "Aberration of light in a Moving Medium," J Phys. A. Gen. Physics, vol. 4, 1971.

24. P. Jackson and R. K. Nixey, "Inertial Frame Error Discovery derives Stellar Aberration and Paradox Free Special Relativity via Huygens Principle," Figure 1. Available: https://www.researchgate.net/publication/ 330508264

25. E. H. Dowdye, Jr., "Time resolved images from the center of the Galaxy appear to counter General Relativity," Astronomical Notes, vol. 328, is. 2, pp. 186-191, 2007. https://onlinelibrary.wiley.com/doi/abs/10.1002/ asna.200510715

26. Yao-Xion Huang, "Reflection and transmission of electromagnetic waves by a dielectric medium moving in an arbitrary direction," Jnl of Applied Physics 76, 2575, 1994. https://aip.scitation.org/doi/pdf/10.1063/ 1.357552 ?class $=$ pdf

27. A. Gjurchinovski and K. Trencevski, "Huygens' construction in a dispersive medium moving at a constant velocity," 2007. Available: https://arxiv.org/abs/0711.0585

28. A. B. Pushkarev et al., "VLBA observations of a rare multiple quasar imaging event caused by refraction in the interstellar medium," A\&AA, vol. 555, A80, 2013. Available: http://arxiv.org/abs/1305.6005

29. P. A. Jackson. "Much Ado About Nothing," FQXi, 2012, p. 7. Available: http://fqxi.org/community/forum/ topic/1330

30. G. H. Kaplan, "The IAU Resolutions on Astronomical Reference Systems," Time Scales, and Earth Rotation Models, USNO, Circular 179, p. 6, 2005. Available: http://aa.usno.navy.mil/publications/docs/Circular_179.pdf

31. K. Brown, Refraction At A Plane Boundary Between Moving Media, Available: https://www.mathpages.com/ $\mathrm{rr} / \mathrm{s} 2-08 / 2-08 . h t m$

32. J. S. Bell, Speakable and Unspeakable in Quantum Mechanics, Cambridge Press, 1987. 1991 ed., ch. 3, p. 27.

33. J. S. Bell, Speakable and Unspeakable in Quantum Mechanics, Cambridge Press, 1987. 1991 ed., ch. 19, p. 175.

34. P. A. Jackson, "Ridiculous Simplicity," FQXi, 2018. Available: https://fqxi.org/community/forum/topic/3012

35. D. A. Traill, "A Fundamental Misunderstanding," FQXi, 2018. Available: https://fqxi.org/community/forum/ topic/3014

36. W.Thomson, (1st Baron Kelvin), Baltimore Lectures on Molecular Dynamics and the Wave Theory of Light. Publication Agency of the Johns Hopkins University, Lecture XVII, 1884. Cambridge Press, C. J. Clay \& Sons, 1904, p.279. https://archive.org/stream/baltimorelecture00kelviala/baltimorelecture00kelviala_djvu.txt Lecture XVII, 23. 